\title{
Application of a Linear Ion Trap/Orbitrap \\ Mass Spectrometer in Metabolite \\ Characterization Studies: Examination \\ of the Human Liver Microsomal Metabolism \\ of the Non-Tricyclic Anti-Depressant \\ Nefazodone Using Data-Dependent \\ Accurate Mass Measurements
}

\author{
Scott M. Peterman and Nicholas Duczak, Jr. \\ Thermo Electron Corporation, Somerset, New Jersey, USA
}

\author{
Amit S. Kalgutkar, Mary E. Lame, and John R. Soglia \\ Pharmacokinetics, Dynamics, and Metabolism Department, Pfizer Global Research and Development, \\ Groton, Connecticut, USA
}

\begin{abstract}
We report herein, facile metabolite identification workflow on the anti-depressant nefazodone, which is derived from accurate mass measurements based on a single run/experimental analysis. A hybrid LTQ/orbitrap mass spectrometer was used to obtain accurate mass full scan MS and MS/MS in a data-dependent fashion to eliminate the reliance on a parent mass list. Initial screening utilized a high mass tolerance $(\sim 10 \mathrm{ppm})$ to filter the full scan MS data for previously reported nefazodone metabolites. The tight mass tolerance reduces or eliminates background chemical noise, dramatically increasing sensitivity for confirming or eliminating the presence of metabolites as well as isobaric forms. The full scan accurate mass analysis of suspected metabolites can be confirmed or refuted using three primary tools: (1) predictive chemical formula and corresponding mass error analysis, (2) rings-plus-double bonds, and (3) accurate mass product ion spectra of parent and suspected metabolites. Accurate mass characterization of the parent ion structure provided the basis for assessing structural assignment for metabolites. Metabolites were also characterized using parent product ion $\mathrm{m} / \mathrm{z}$ values to filter all tandem mass spectra for identification of precursor ions yielding similar product ions. Identified metabolite parent masses were subjected to chemical formula calculator based on accurate mass as well as bond saturation. Further analysis of potential nefazodone metabolites was executed using accurate mass product ion spectra. Reported mass measurement errors for all full scan MS and MS/MS spectra was $<3 \mathrm{ppm}$, regardless of relative ion abundance, which enabled the use of predictive software in determining product ion structure. The ability to conduct biotransformation profiling via tandem mass spectrometry coupled with accurate mass measurements, all in a single experimental run, is clearly one of the most attractive features of this methodology. (J Am Soc Mass Spectrom 2006, 17, 363-375) (C 2006 American Society for Mass Spectrometry
\end{abstract}

$\mathrm{M}$ etabolite identification constitutes an important paradigm at various stages of drug discovery and development. In the early discovery stage, it is crucial to identify metabolic soft spots in lead chemical series to provide feedback to medicinal chemists for further optimization of the lead com-

Published online January 25, 2006

Address reprint requests to Dr. A. Kalgutkar, Pharmacokinetics, Dynamics, and Metabolism Department, Pfizer Global Research and Development, Eastern Point Road, Groton, CT 06340, USA. E-mail: amit.kalgutkar@pfizer.com pounds. Metabolite identification studies at this stage are usually designed to avoid metabolic instability that may lead to extensive first pass metabolism and poor oral bioactivity [1]. In addition, early metabolite identification studies are also extensively utilized to minimize potential safety concerns that may arise from bioactivation of lead compounds to electrophilic reactive intermediates [2]. For a drug candidate in preclinical development an early understanding of the in vivo metabolic fate is essential since metabolites can contribute to pharmacologic action or possess toxicological conse- 
quences independent of the parent. Owing to its selectivity, sensitivity, and speed of analysis with minimal sample processing, liquid chromatography tandem mass spectrometry (LC-MS/MS) has become the method of choice for metabolite identification in the fast paced environment of drug discovery/development [3]. Crude extracts from complex biological matrices can be subject to metabolite identification studies directly on a LC-MS/MS. Complex metabolite profiles can be chromatographically separated on an HPLC column and full scan MS and product ion scan MS/MS data generated on-line. The molecular weight of metabolites and localization of biotransformation sites can be elucidated based on interpretation of MS/MS data obtained by collision-induced dissociation (CID) of the parent structure and the various metabolites.

Structural elucidation of metabolites is not always straightforward, particularly in cases of complex rearrangement of chemical architecture leading to unique metabolites whose structure(s) cannot be elucidated by the usual LC-MS/MS methodology [3-5]. Likewise, the presence of metabolites with isobaric molecular ions and/or competing fragment ions in parent drug MS confound precise determination of fragmentation pattern in the parent drug and its metabolites $[3,6]$. In the latter case, competing fragmentation pathways in parent and metabolite mass spectra can afford product ions separated by as little as 0.04 to $0.1 \mathrm{Da}$. Using low-resolution mass spectrometry, these minute differences in observed fragment ion masses often preclude an unambiguous elucidation of fragment ion structure and consequently metabolite structure. In some instances, the ensuing dogma may be resolved via synthesis of stable isotope (e.g., ${ }^{13} \mathrm{C},{ }^{2} \mathrm{H},{ }^{15} \mathrm{~N}$ ) derivatives of the parent molecule [7]. However, such strategies may hinder progress in a fast paced drug discovery environment, since additional efforts/resources must be dedicated towards the synthesis of stable isotope analogs, which can often require multistep synthesis. Supplementing low-resolution MS with accurate mass measurements in metabolite identification studies can provide valuable information in distinguishing isobaric molecular ions and provide unequivocal proof of correct fragmentation patterns, resulting in a significant increase in confidence towards predictions of metabolite structure [8,9]. While time-of-flight (TOF) instrumentation is often used to acquire accurate mass information $[10,11]$, the methodology requires numerous LC runs to acquire accurate mass on multiple product ions and can be potentially viewed as time and cost prohibitive in a typical drug discovery scenario. Difficulties associated with using TOF technology are attributed to a dependence on internal calibrants or lock masses for obtaining high mass accuracy measurements or recalibration offline. In addition, mass accuracy may be compromised at extremely low relative ion intensities resulting in missed diagnostic ions that can be used to further identify metabolites.

Recently, the combination of Orbitrap technology with a linear ion trap has been shown to enable fast, sensitive and reliable detection and identification of small molecules regardless of relative ion abundance $[12,13]$. The orbitrap mass analyzer employs the trapping of pulsed ion beams in an electrostatic quadrologarithmic field. This field is created between an axial central electrode and a coaxial outer electrode. Stable ion trajectories combine rotation around the central electrode with harmonic oscillations along it. The frequencies of axial oscillations and hence mass-to-charge ratios of ions are obtained using fast Fourier transform of the image current detected on the two split halves of the outer electrode. The hybrid linear ion trap/orbitrap mass spectrometer is related to the Finnigan LTQ FT in combining fast scan speeds and high mass accuracy measurements (within a few ppm) for precursor and product ions on an LC timescale which is critical for metabolic profiling. Furthermore, external calibration can be used to obtain high mass accuracy ( $<3 \mathrm{ppm}$ ) of all measured spectra resulting in a simplified experimental protocol.

As a demonstration of the capability of this de Novo technology, we re-examined the metabolic pathways of the non-tricyclic anti-depressant drug nefazodone in NADPH-supplemented human liver microsomes utilizing accurate mass measurements of precursor and MS/MS product ions for the parent drug and its metabolites [14-17]. Data-dependent MS/MS analyses were conducted on a hybrid linear ion trap/orbitrap mass spectrometer that enabled high-resolution/high mass accuracy measurements on precursors and product ions regardless of relative ion abundance and on a LC-timescale [12, 13, 18]. Furthermore, increased mass accuracy measurements helped in assigning fragment ion structure for all anticipated and novel metabolites of the drug.

\section{Experimental}

\section{Materials}

Nefazodone hydrochloride and NADPH were purchased from Sigma-Aldrich (St. Louis, MO). Human liver microsomes pooled from 53 individual donors were purchased from BD Gentest (Woburn, MA).

\section{Microsomal Incubations}

Stock solutions of nefazodone were prepared in methanol. The final concentration of methanol in the incubation media was $0.2 \%$ ( $\mathrm{vol} / \mathrm{vol})$. Incubations were carried out at $37^{\circ} \mathrm{C}$ for $60 \mathrm{~min}$ in a shaking water bath. The incubation volume was $1 \mathrm{~mL}$ and consisted of the following: $0.1 \mathrm{M}$ potassium phosphate buffer ( $\mathrm{pH} 7.4)$, human liver microsomes (P450 concentration $=0.5$ $\mu \mathrm{M}), \mathrm{NADPH}(1.2 \mathrm{mM})$, and nefazodone $(10 \mu \mathrm{M})$. The 
reaction mixture was prewarmed at $37^{\circ} \mathrm{C}$ for $2 \mathrm{~min}$ before adding NADPH and incubations were terminated by the addition of ice-cold acetonitrile $(1 \mathrm{~mL})$. The solutions were centrifuged (3000 $g$ for $15 \mathrm{~min}$ ) [16], and the supernatants were dried under a steady nitrogen stream. The residue was reconstituted with mobile phase and analyzed for metabolite formation.

\section{LC-MS/MS Assay for Identification of Nefazodone Metabolites}

Metabolites were identified by a Finnigan LTQ orbitrap mass spectrometer (Thermo Electron, Bremen, Germany) and all ion acquisition was performed using the orbitrap mass spectrometer. The LC system consisted of a Hypersil Gold $(1.9 \mu \mathrm{M} \mathrm{C18} 50 \times 2.1 \mathrm{~mm})$ column, a Finnigan Surveyor LC system comprising of an HPLC with a built-in degasser, autosampler, and a Finnigan LTQ orbitrap mass spectrometer. A constant flow rate of $0.27 \mathrm{~mL} / \mathrm{min}$ was used with a gradient from $3-95 \% \mathrm{~B}$ in $35 \mathrm{~min}$ before column re-equilibration. Solvent $\mathrm{A}$ was $10 \mathrm{mM}$ ammonium formate $(0.1 \%$ formic acid) and Solvent B was acetonitrile (100\%). The scan event cycle used a full scan mass spectrum at a resolving power of 15,000 (at $\mathrm{m} / \mathrm{z} 400$ ) and three corresponding data-dependent MS/MS events acquired at a resolving power of 7500. The three most intense ions detected during full scan MS triggered data dependent scanning. Data dependent scanning was performed without the use of a parent ion list. The microscan count for full and MS/MS scan events were set to unity and a repeat count for dynamic exclusion was set to three for verification. MS/MS activation parameters used an isolation width of $2 \mathrm{Da}$, normalized collision energy of $35 \%$, and an activation time of 30 milliseconds. Mass accuracy was determined from an external calibration that was performed the prior day.

\section{Data Interpretation}

All data were processed using Qual Browser (Thermo Electron, San Jose, CA), which provides accurate mass thresholds to filter data. In addition, chemical formula calculator was used to provide chemical formula and saturation values (ring-plus-doublebonds [RDB]) for product ions of nefazodone and its metabolites [19]. Fragmentation patterns were generated using Mass Frontier 4.0 (Thermo Electron) [20, 21]. Predictive fragmentation pattern was derived from plausible protonation sites, subsequent isomerization and even electron species, and bond saturation (i.e., RDB). Comparisons between theoretical and experimental product ion spectra further aided in the identification of metabolite structures and site(s) of biotransformation with the parent drug.

\section{Results and Discussion}

Scheme 1 depicts the observed molecular ions $[\mathrm{M}+\mathrm{H}]^{+}$, retention times $\left[R_{\mathrm{t}}, \mathrm{min}\right]$, and calculated $\mathrm{RDB}$ values for nefazodone and its metabolites. Figure 1 shows the total ion chromatogram of a NADPH-supplemented human liver microsomal incubation of nefazodone $(10 \mu \mathrm{M})$ that is baseline subtracted from control experiments lacking NADPH co-factor. In addition to unmetabolized nefazodone, which eluted at $13.36 \mathrm{~min}$, there are numerous lower intensity peaks, which can be attributed to metabolites derived from the oxidation of nefazodone. Metabolite profiling studies were initiated following a thorough investigation of the mass spectral fragmentation pattern of the nefazodone parent molecule considering that determination of precise fragmentation pattern of the parent molecule enhances confidence towards predicting biotransformation site(s) on the corresponding metabolite product ion.

Figure 2 shows a product ion spectrum of nefazodone resulting from one total scan comprised of a single microscan. The total time for product ion spectrum acquisition was 340 milliseconds. The product ion spectrum is dominated by the fragment ion $\mathrm{m} / \mathrm{z} 274$ (measured ion intensity $\sim 2.1 \times \mathrm{e}^{07}$ counts and mass error of $-1.4 \mathrm{ppm}$ ) corresponding to the phenoxyethyl5 -ethyltriazolone propyl motif. In addition, there are several low intensity product ions that are consistent with fragmentation spectra predicted from Mass Frontier 4.0. The structures and corresponding mass errors for these low intensity fragment ions are presented in Figure 2. It is interesting to point out that despite large differences in measured ion intensity between product ions, the measured mass accuracy are quite comparable. For example, there is ca. a 100-fold difference between the measured ion intensities of the product ions at $\mathrm{m} / \mathrm{z}$ $140\left(5.7 \times \mathrm{e}^{05}\right)$ and at $m / z 274\left(2.1 \times \mathrm{e}^{07}\right)$, yet the differences in the measured mass accuracy are fairly comparable ( -2.8 and $-1.4 \mathrm{ppm}$ for the product ions at $\mathrm{m} / \mathrm{z} 140$ and 274, respectively). In addition, the inset in Figure 2 shows the lowest intensity product ions detected from the single product ion spectrum. The product ion at $\mathrm{m} / \mathrm{z} 197$ has a measured ion intensity of $8.2 \times$ $\mathrm{e}^{03}$ and a measured mass error of $4.5 \mathrm{ppm}$. This represents almost a 3000-fold difference in measured ion intensities without a significant compromise in product ion assignment.

The structure of nefazodone presents a challenge for interpretation of the product ion spectra due to isobaric product ions that can be predicted to arise, based on site of protonation and subsequent bond scission. For example, the $\mathrm{N}$-alkylsubstituted nitrogen atom in the piperazine ring (Figure 2) provides a basic center for charge retention that could potentially lead to multiple bond scissions before observed product ion formation. The competing product ions may have isobaric massto-charge values separated by $0.05 \mathrm{Da}$ (ca. $200 \mathrm{ppm}$ ), and thus, one would need to rely on accurate mass measurements for correct prediction of fragment ion 


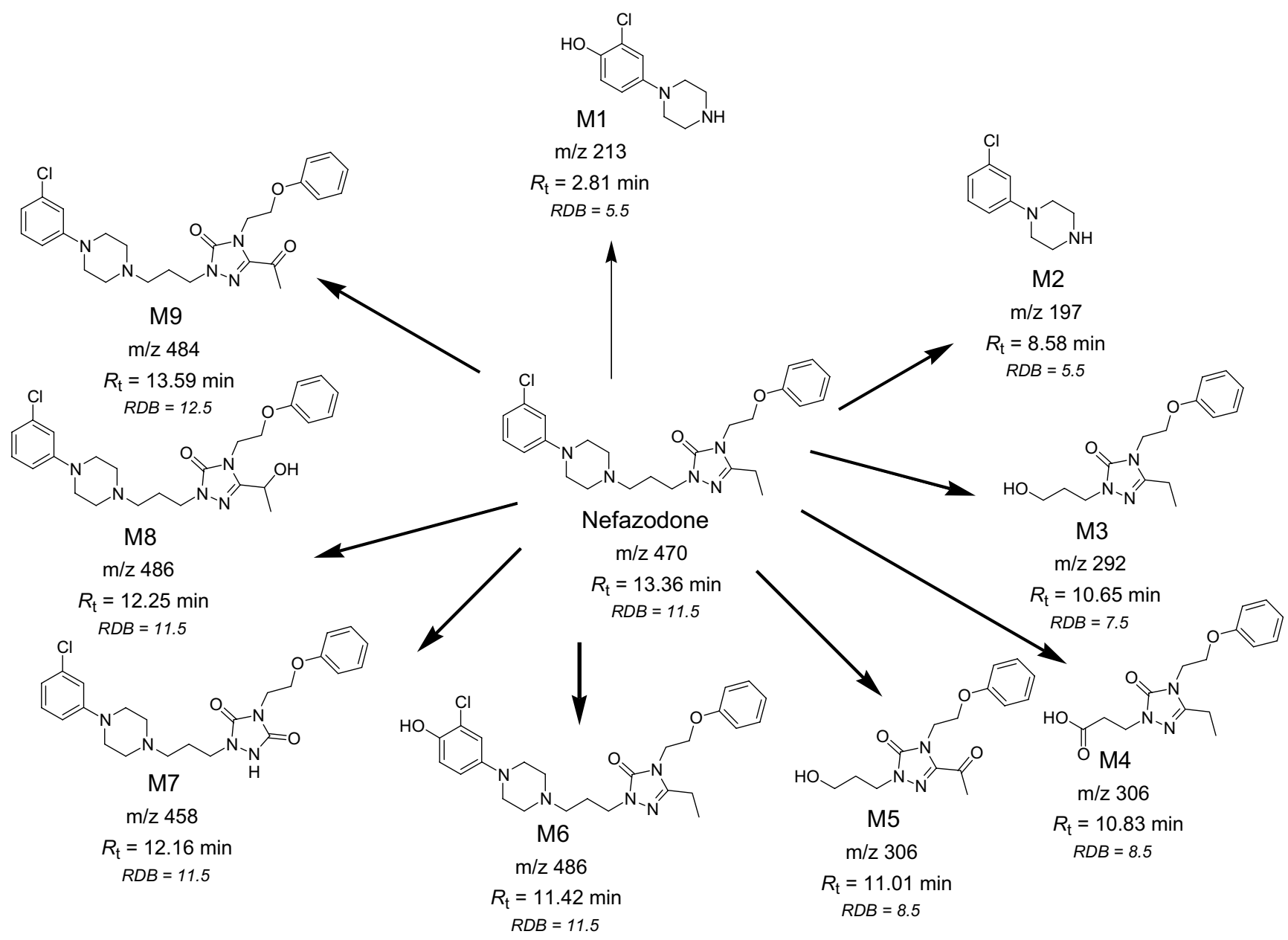

Scheme 1. Metabolic pathways of the anti-depressant nefazodone in humans.

structure. Figure 3 depicts the competing pathways predicted by Mass Frontier 4.0 that would give rise to the product ion at $\mathrm{m} / \mathrm{z} 180$. Assuming protonation is occurring on the piperazine nitrogen shown in Figure 3, the product ion of the "left" side of the nefazodone structure would possess a mass of 180.0575 Da (Figure 3, Pathway A) while the product ion that arises from the right hand side of the molecule will possess a mass of 180.1131 Da (Figure 3, Pathway B). The measured mass of 180.1128 unambiguously proves that cleavage on the right hand side of the nefazodone structure is the most likely route to the $\mathrm{m} / \mathrm{z} 180$ product ion given that the resulting mass error is only 2 ppm compared to 307 ppm for the product ion resulting from cleavage on the left hand portion of nefazodone (Table 1). In addition to the product ion at $m / z 180$, nefazodone fragmentation affords other product ions that can be assigned multiple structures that differ by less than $0.1 \mathrm{Da}$. Table 1 lists the product ions observed following nefazodone collisional activation. The experimentally observed mass-to-charge values are listed with the predicted product ions from Mass Frontier 4.0. Note that five out of the eight product ions have predicted product ions with charge retention on each side of the nefazodone structure, but with accurate mass measurements, fragmentation pattern is accurately determined in each of these cases.

Figure 4 depicts a series of extracted ion chromatograms for various nefazodone metabolites. The observed molecular ions and fragment ion spectra of these metabolites are consistent with those previously reported in the in vivo and in vitro metabolism studies on nefazodone in humans [14-17]. Individual metabolite ions were initially extracted from the total ion chromatogram using $\mathrm{m} / \mathrm{z}$ values for nefazodone metabolites from previously published reports. A mass threshold of $10 \mathrm{ppm}$ was used to determine "real" metabolite signal. The benefits of using a high mass tolerance dramatically improved signal-to-noise ratio and allowed an easy determination of the presence of isomeric metabolites as well as metabolites which were not predicted from Mass Frontier 4.0 and/or theoretical reasoning. Furthermore, by reducing the background signal from each extracted ion chromatographic trace, a difference in signal intensity of ca. 2800 times is observed between the greatest integrated peak area for the nefazodone parent ion and the minor peak for the metabolite with $\mathrm{m} / \mathrm{z}$ at $484($ mass error $=1.7 \mathrm{ppm})$ that eluted at 12.9 $\mathrm{min}$. The addition of $\mathrm{m} / \mathrm{z} 14$ to the nefazodone parent 


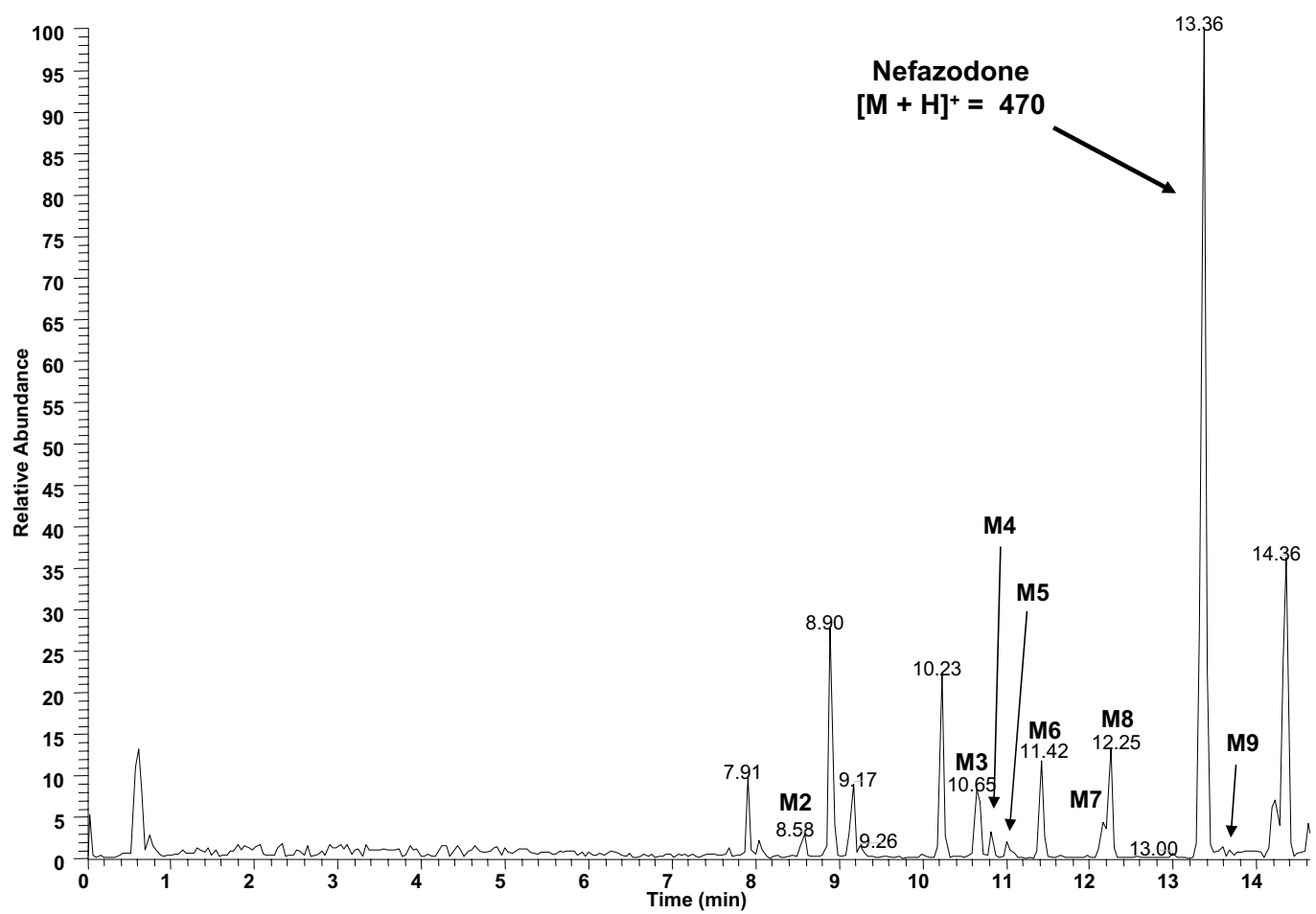

Figure 1. Base peak chromatogram of a NADPH-supplemented human liver microsomal incubation of nefazodone $(10 \mu \mathrm{M})$ as described under Materials and Methods.

molecular weight is consistent with introduction of a carbonyl functionality within the molecule and this metabolite appears to be an isomer of M9. In addition to this set of isomeric metabolites of nefazodone, the extracted ion chromatographic traces also indicated the possible presence of isomeric metabolites for $\mathrm{m} / \mathrm{z} 306$ (Figure 4e) and $m / z 486$ (Figure 4f).

Figure 5 shows comparative product ion spectra for the isomeric monohydroxy metabolites of nefazodone $(\mathrm{m} / \mathrm{z} 486)$, which elute at 11.42 (Figure $5 \mathrm{a}$ ) and $12.25 \mathrm{~min}$

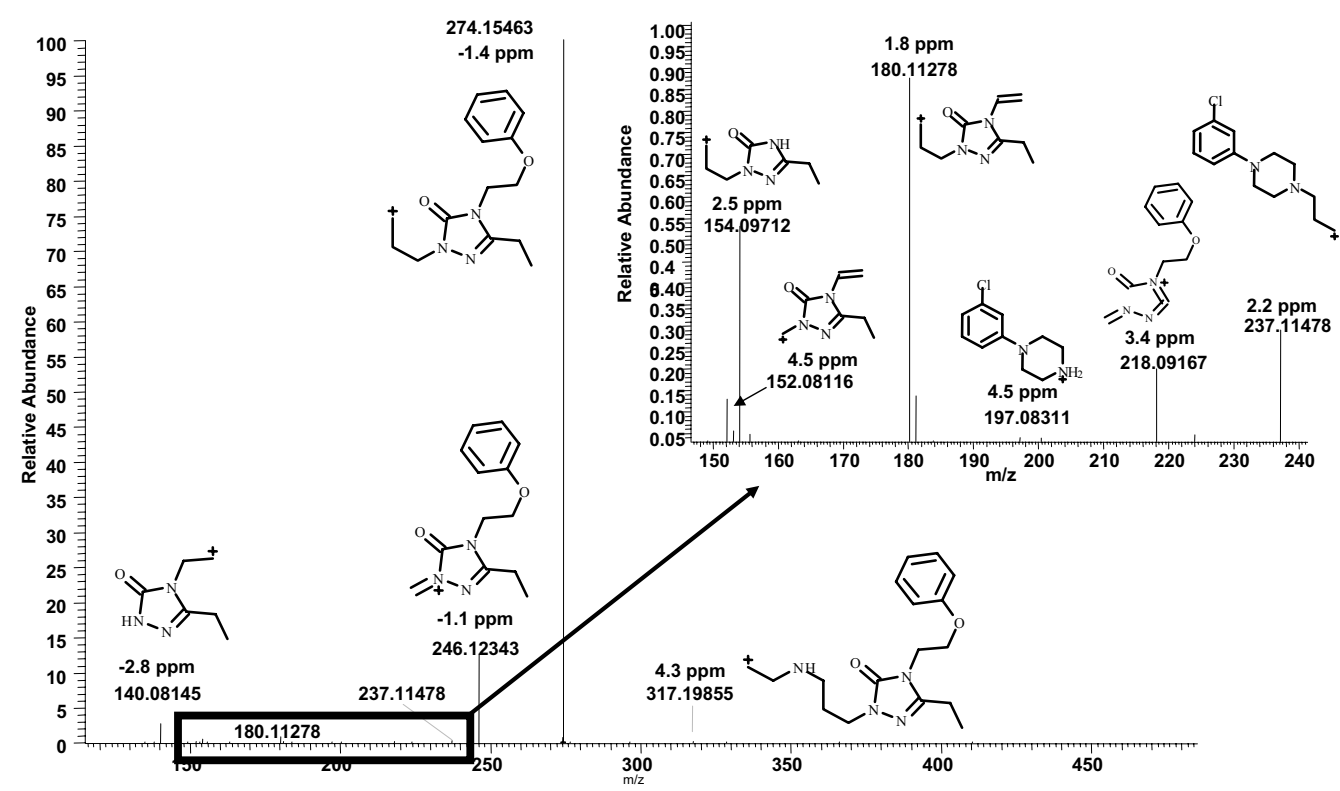

Figure 2. Full scan accurate mass spectrum of nefazodone $(\mathrm{m} / \mathrm{z} 470)$ acquired in the Orbitrap mass spectrometer using external mass calibration. The expanded mass range shown is shown for low intensity product ions. Product ion assignment resulted from predictive fragmentation using Mass Frontier 4.0. 
(Figure 5b), respectively. The mass accuracy associated with each fragment ion resulted from comparison of experimental values with predicted structures from Mass Frontier 4.0. The spectrum shows the 20-fold magnified region covering the mass range of 135 to 265 Da. The product ion spectra (Figure 5a) of the monohydroxynefazodone metabolite at a $R_{\mathrm{t}}$ of 11.42 min reveals a single fragment ion at $\mathrm{m} / \mathrm{z} 253$ representing an addition of mass 16 to the fragment ion at $\mathrm{m} / z 237$ in the nefazodone parent ion spectra (see Figure 2) implicating the 3-chlorophenylpiperazine ring as the site of hydroxylation. In contrast, the product ion spectrum (Figure $5 b$ ) of the monohydroxylated nefazodone metabolite at an $R_{\mathrm{t}}$ of 12.22 min depicts diagnostic fragment ions at $m / z 152,170,209$, and 262. The mass error for the product ion at $\mathrm{m} / \mathrm{z} 209$, which corresponds to the 3 -chlorophenylpiperazine ring region within nefazodone, is $-0.3 \mathrm{ppm}$. The fragment ions at $\mathrm{m} / \mathrm{z} 170$ (mass

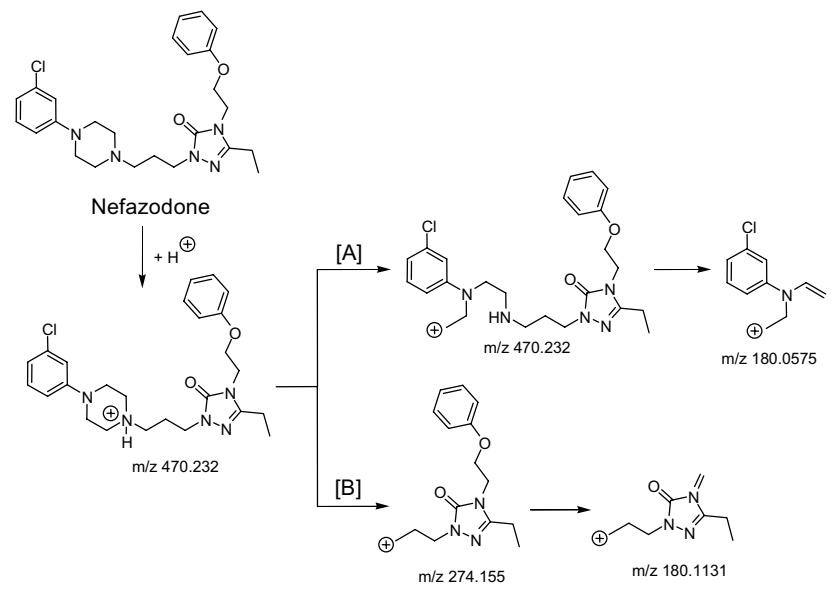

Figure 3. Competitive reaction schemes for the formation of the $\mathrm{m} / \mathrm{z} 180$ product ion resulting from collisional activation of nefazodone. The reaction mechanisms are predicted using Mass Frontier 4.0.

Table 1. List of experimentally observed mass measurements of the nefazodone product ions following CID and the theoretically predicted mass-to-charge values for two plausible product ions obtained from Mass Frontier 4.0

\begin{tabular}{ccccr}
\hline $\begin{array}{c}\text { Corresponding } \\
\text { mass error }(\mathrm{ppm})\end{array}$ & $\begin{array}{c}\text { Theoretical mass } \\
\text { (left side fragment) }\end{array}$ & $\begin{array}{c}\text { Experimental mass } \\
\text { (measured intensity) }\end{array}$ & $\begin{array}{c}\text { Theoretical mass } \\
\text { (right side fragment) }\end{array}$ & $\begin{array}{c}\text { Corresponding } \\
\text { mass error (ppm) }\end{array}$ \\
\hline \hline 394.93 & 140.02615 & $140.08145(5.7 \mathrm{e} 5)$ & 140.08184 & -2.78 \\
395.12 & 154.04180 & $154.09712(1.1 \mathrm{e} 5)$ & 154.09749 & -2.40 \\
307.29 & 180.05745 & $180.11278(1.8 \mathrm{e} 5)$ & 180.11314 & -2.00 \\
-4.52 & 197.08400 & $197.08311(8.2 \mathrm{e} 3)$ & 197.13969 & -287.00 \\
N/A & N/A & $218.09167(3.6 \mathrm{e} 4)$ & 218.09240 & -3.35 \\
-2.19 & 237.11530 & $237.11478(5.4 \mathrm{e} 4)$ & 237.17099 & -237.00 \\
N/A & N/A & $246.12343(2.6 \mathrm{e} 6)$ & 246.12370 & -1.10 \\
N/A & N/A & $274.15463(2.1 \mathrm{e} 7)$ & 274.15500 & -1.35 \\
\hline
\end{tabular}

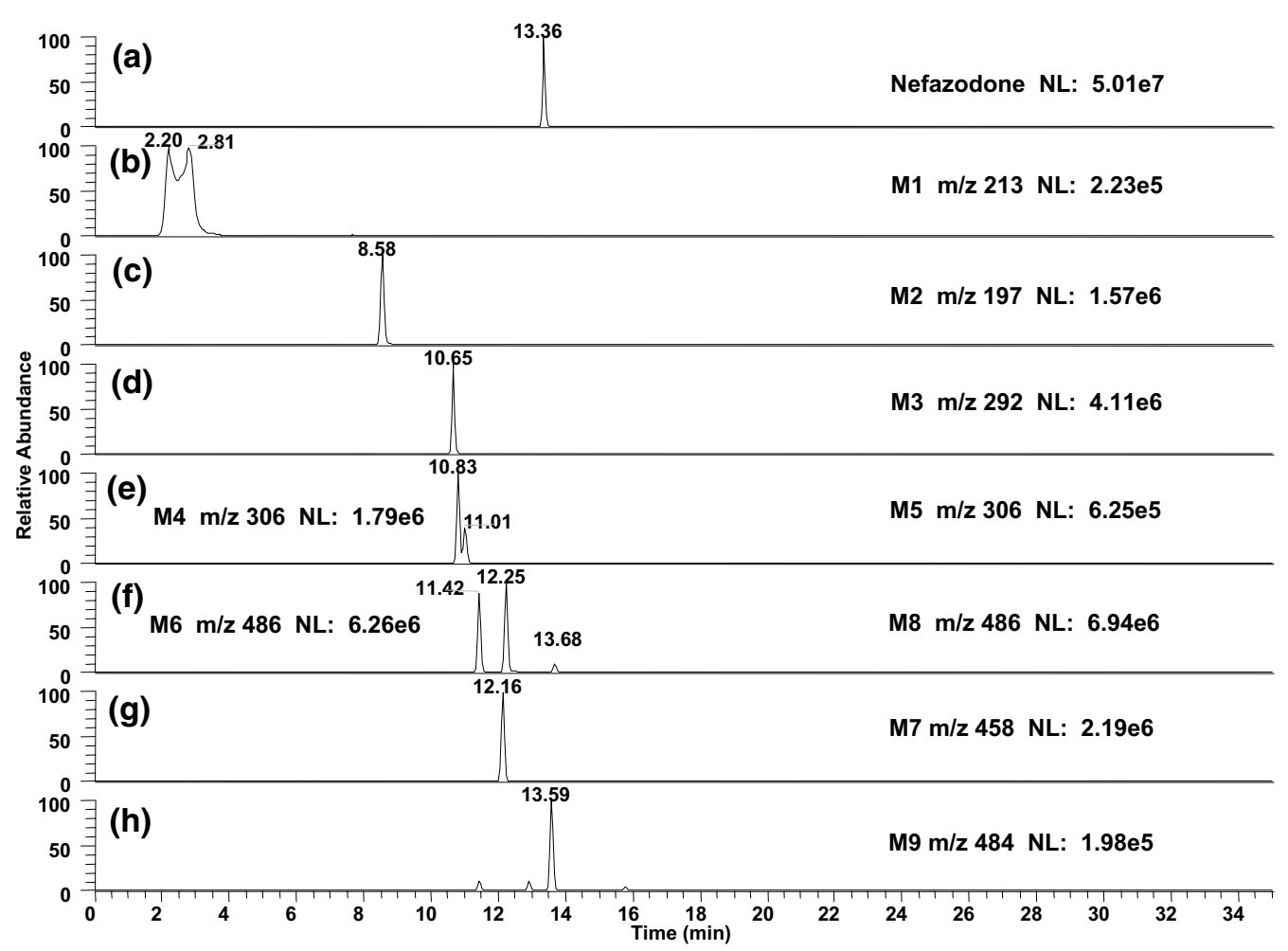

Figure 4. Extracted ion chromatograms in the positive ionization mode for nefazodone and eight of its primary metabolites. A 10 ppm mass tolerance was used to filter the full scan MS data. 


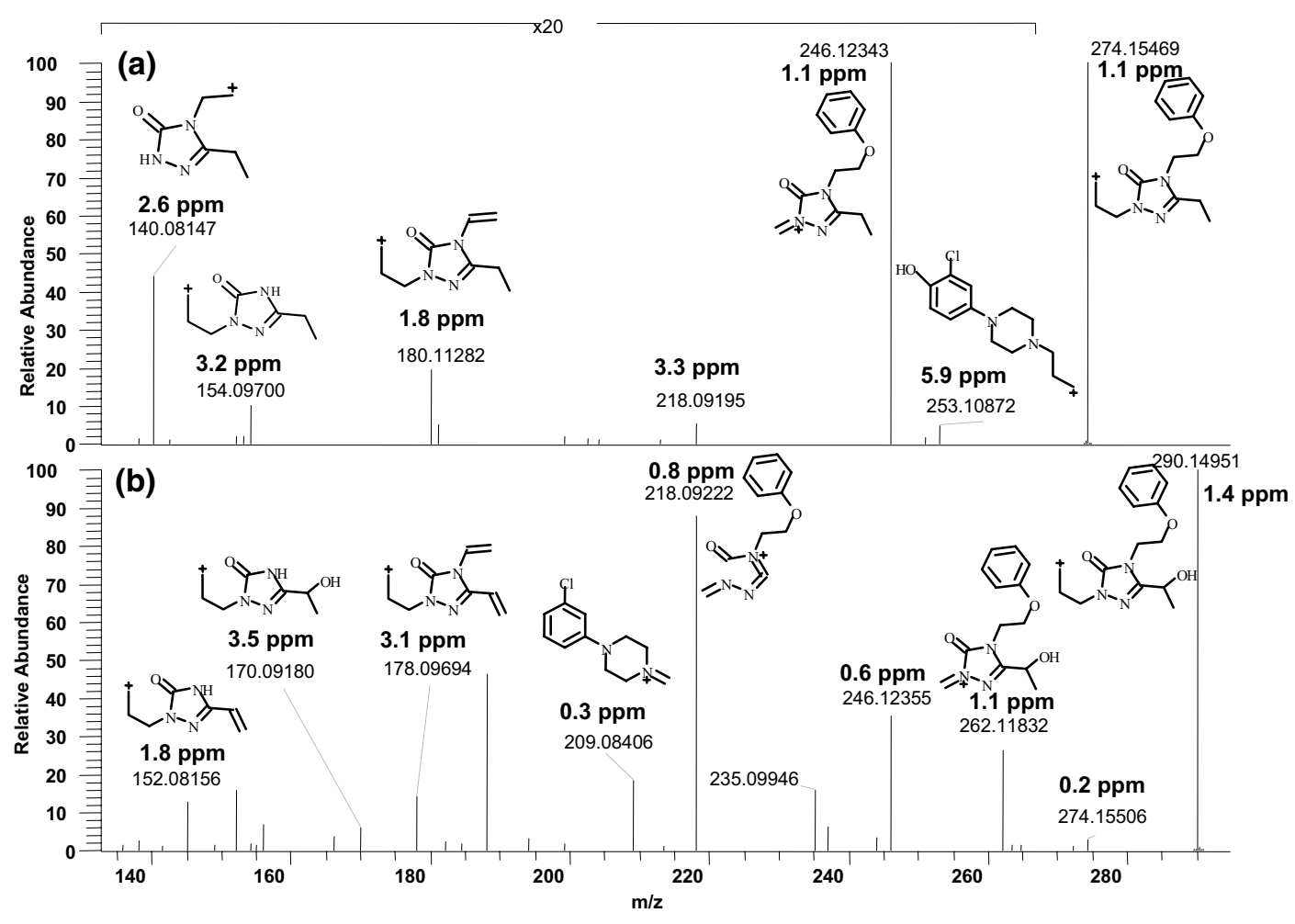

Figure 5. Comparison of the MS/MS spectra of monohydroxylated nefazodone metabolites M6 $\left(R_{t}\right.$ $=11.42 \mathrm{~min})$ and $\mathrm{M} 8\left(\mathrm{R}_{\mathrm{t}}=12.25 \mathrm{~min}\right)$.

error $=3.5 \mathrm{ppm})$ and $262(1.1 \mathrm{ppm})$, which represent the additions of mass 16 to the fragment ions at $\mathrm{m} / \mathrm{z} 154$ and $\mathrm{m} / \mathrm{z} 246$, respectively, in the product ion spectrum of nefazodone implicates the ethyl group in the 5-ethyltriazolone motif as the site of monohydroxylation. Overall, the structures of these metabolites M6 and M8 (see Scheme 1) are consistent with those reported in previous studies [14-17].

Accurate mass filtering of the full scan mass spectral data also revealed the possibility of a second $\mathrm{m} / \mathrm{z} 306$ metabolite (M5 in Scheme 1), which eluted at $11.01 \mathrm{~min}$ and appears to be an isomer of the previously characterized metabolite M4 (see Scheme 1) that elutes at 10.83 min. Both metabolites are derived from downstream metabolism of the $\mathrm{N}$-dealkylated metabolite M3 that has lost the 3-chlorophenylpiperazine ring. Data-dependent MS/MS spectra for each of the $\mathrm{m} / \mathrm{z} 306$ metabolites are presented in Figure 6. The resulting product ion spectra for M4 (Figure 6a) contain the product ions at $\mathrm{m} / \mathrm{z} 140$ (mass error $=2.6 \mathrm{ppm}$ ) and 246 (mass error $=$ $2.0 \mathrm{ppm}$ ), which are also present in the mass spectrum of nefazodone. These ions along with the fragment ion at $\mathrm{m} / \mathrm{z} 288$ (representative of the acylium ion derived from carboxylic acid fragmentation) are in excellent agreement with the previously reported mass spectrum of M4 [14, 16]. The previously undisclosed $\mathrm{m} / \mathrm{z} 306$ metabolite eluting at $11.01 \mathrm{~min}$ shows additional diagnostic product ions that can be utilized to gain insight into its structure (Figure $6 \mathrm{~b}$ ). Specifically the fragment ions at $\mathrm{m} / \mathrm{z} 168$ and 194 provide insight into the two distinct sites of oxidative metabolism as shown in the proposed structure for M5 in Scheme 1. The mass error associated with each of the unique fragment ions is 1.1 and $1.9 \mathrm{ppm}$. The degree of bond saturation based on the Mass Frontier derived chemical formula is calculated to be 4.5 and 5.5, which agrees with the predicted product ion structure. We propose that M5 arises from the P4503A4-mediated N-dealkylation of M9.

Further examination of the LC-MS/MS analysis of nefazodone microsomal incubation was conducted to probe for the formation of additional and perhaps unanticipated metabolites. The fragment ions identified in the mass spectrum of nefazodone were also utilized in extracting metabolite ions from all data-dependent MS/MS spectra, thus providing an alternate tool for data analysis. Figure 7 shows extracted ion chromatograms for the product ions at $\mathrm{m} / \mathrm{z}$ values of 140.0818 (Figure 7a) and 274.15,500 (Figure $7 b$ ) from MS/MS spectra obtained from nefazodone microsomal incubations. The mass tolerance used for the extracted ion chromatograms was $10 \mathrm{ppm}$ and the precursor ion yielding the selected product ions are listed at the top of each peak. It is worth commenting on the selectivity displayed in this analysis when using a tight mass tolerance. For example, Figure $7 \mathrm{a}$ shows predominant peaks at retention times $8.91,10.67,11.43$, and 13.38 $\mathrm{min}$. The baseline in between each of the major peaks is magnified 20-fold. Inspection of the magnified region reflects the degree of selectivity considering that additional metabolite peaks identified in Figure $7 \mathrm{~b}$ (metab- 

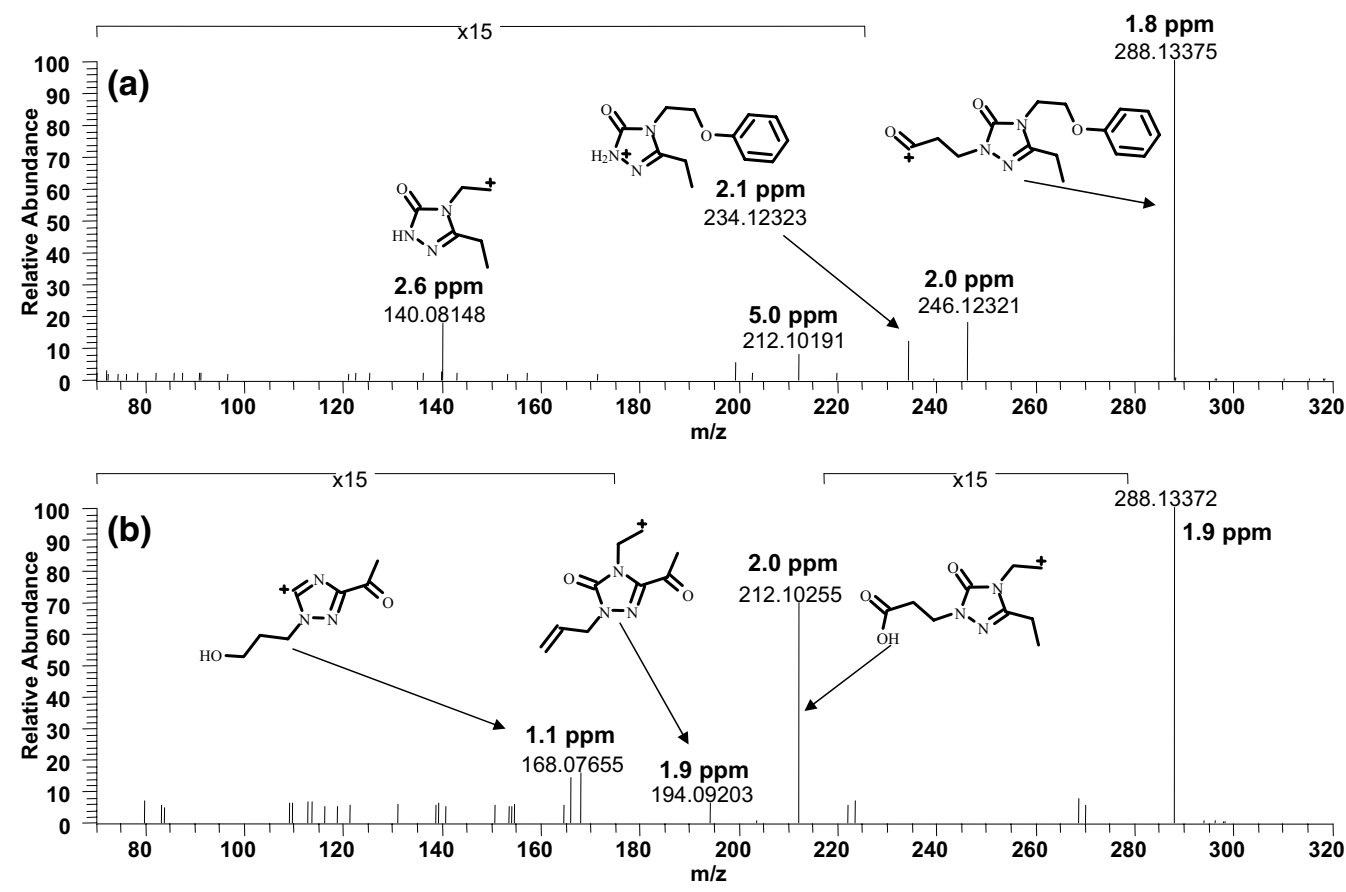

Figure 6. Comparison of the MS/MS spectra for the isomeric $m / z 306$ metabolites M4 $\left(R_{t}=10.83\right.$ $\mathrm{min})$ and $\mathrm{M} 5\left(\mathrm{R}_{\mathrm{t}}=11.01 \mathrm{~min}\right)$.

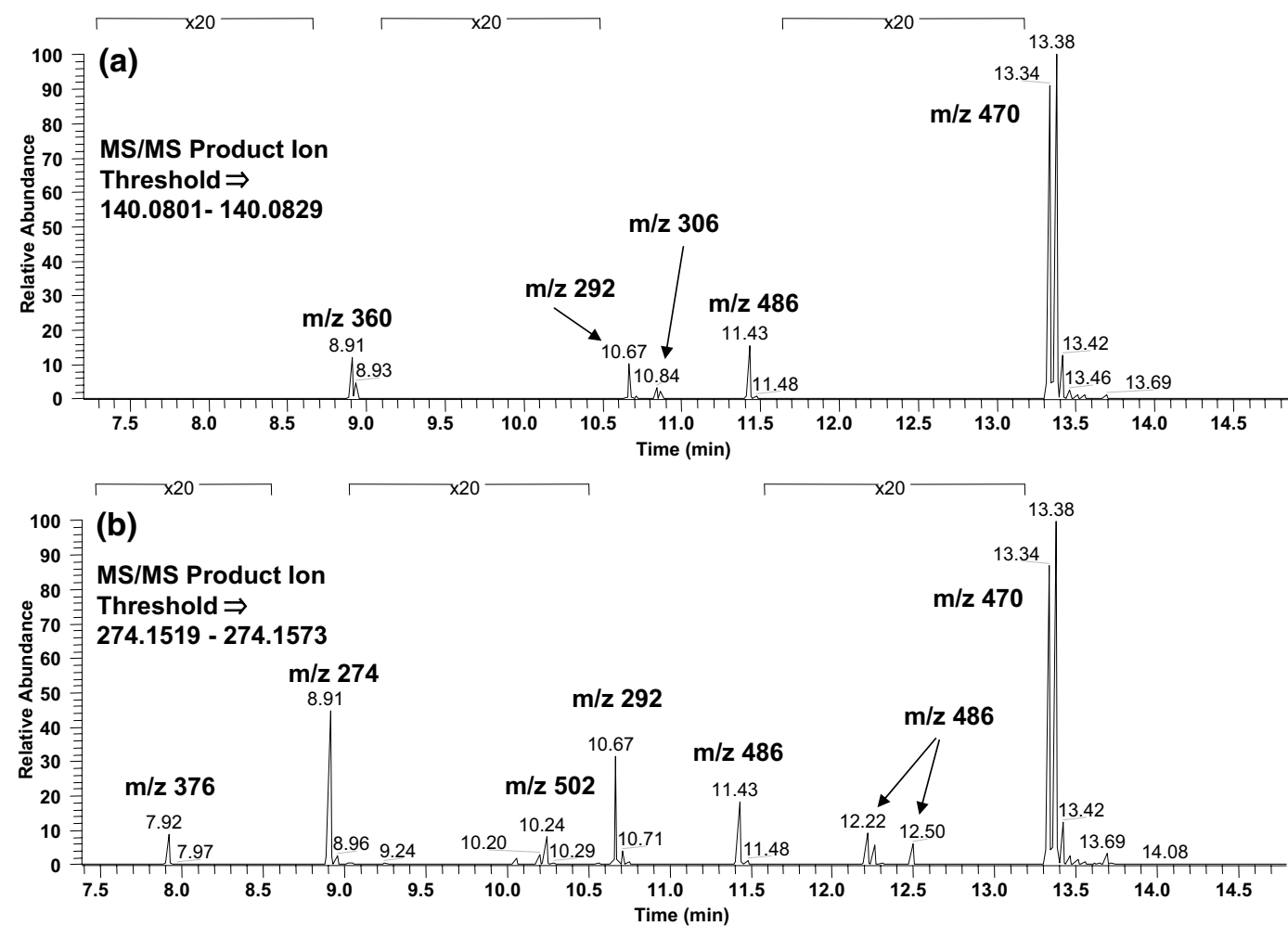

Figure 7. Extracted ion chromatograms for MS/MS product ions from the nefazodone parent drug. Masses labeled indicate the precursor mass isolated for collisional activation. The mass threshold used for chromatographic reconstruction is $10 \mathrm{ppm}$. 


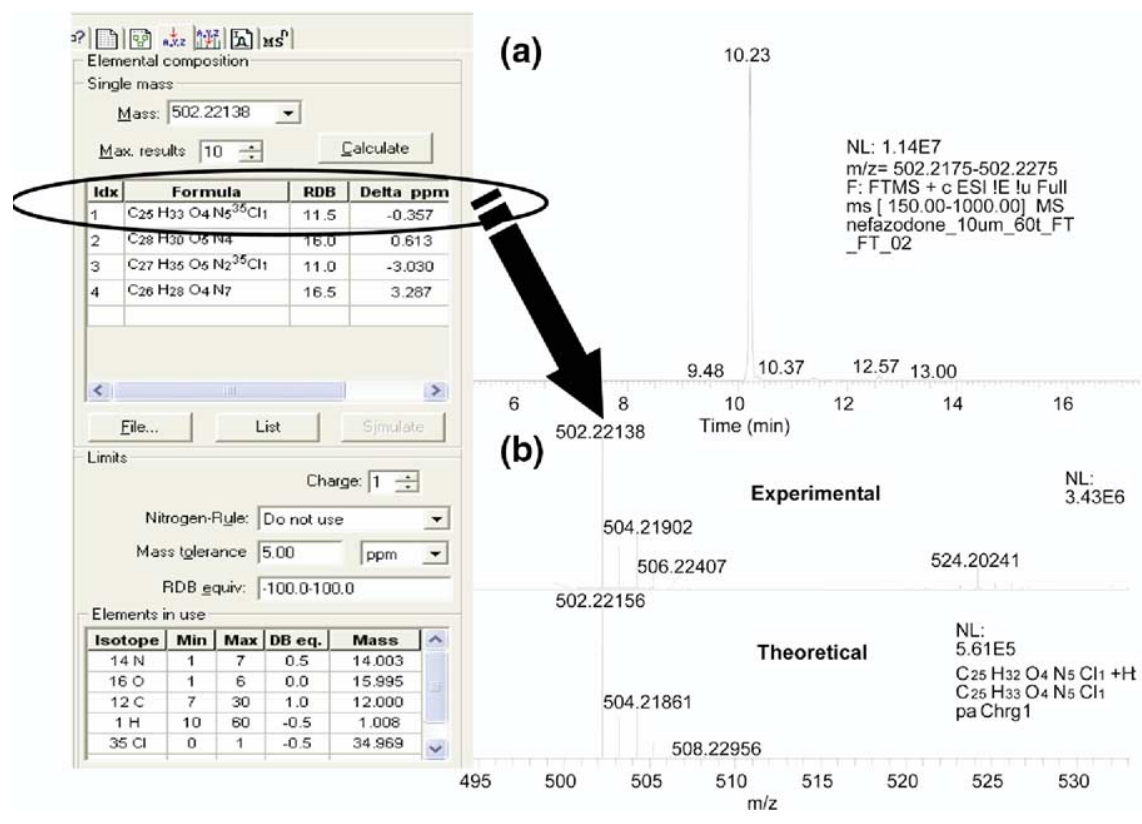

Figure 8. Extracted ion chromatogram for the dihydroxynefazodone metabolite M10 and the corresponding full scan MS. The screen capture from Qual Browser shows the predicted chemical formula and theoretical mass-to-charge value and isotopic distribution.

olites at $\mathrm{m} / \mathrm{z} 376$ and 502 with a retention time of 7.92 and $10.24 \mathrm{~min}$, respectively) are not found in Figure 7a. The use of the linear trap/orbitrap hybrid arrangement thus enables detection of all product ions within a wide mass spectral window as opposed to SRM analysis employed by triple quadrupole mass spectrometers. Therefore, a more thorough interrogation in a postacquisition method can be done by probing the resulting data for evidence of additional metabolites via different product ions from either the parent drug or previously characterized metabolites of the parent drug. In addition, the high mass accuracy data acquisition enables a selective and sensitive means of filtering the resulting product ion spectra. Figure 7 underscores the utility of this approach showing identification of potential metabolites using only two nefazodone product ions. Many more product ions could be used in an exhaustive searching algorithm to thoroughly process the data for identification of as many metabolites as would be deemed necessary.

Further structural analysis of the metabolites identified via the accurate mass product ion filtering was also examined using Qual Browser capability of this instrumentation. Using the elemental composition and ranges of the nefazodone parent structure, each metabolite was matched with a predicted chemical formula and RDB value. Predicted isotopic distribution was also compared to the experimentally determined ratios. An example of the described strategy is shown in Figure 8. Figure 8a shows an extracted ion chromatogram for the $\mathrm{m} / \mathrm{z} 502$ ion filtered using a mass tolerance of $10 \mathrm{ppm}$ and reveals only one elution peak. The observed $\mathrm{m} / \mathrm{z}$ value of 502.22,138 was fitted with potential elemental compositions showed in the bottom left range from the
Qual Browser screen-capture. The 5 ppm mass tolerance was used for prediction of a singly charged species and the RDB constraint was widened to accommodate values ranging from -100 to 100 . This range is for demonstration purposes and can be reduced further to better fit the parent and/or metabolite structure. As shown in the screen capture image in Figure 8, the formulas predict four possible elemental compositions for the observed $\mathrm{m} / \mathrm{z}$ value. The first two formulae are associated with mass errors less than $1 \mathrm{ppm}$ while the latter two formulas have mass errors around $3 \mathrm{ppm}$. Examination of the isotopic distribution indicates the presence of one chlorine atom, which eliminates the second and fourth formulae. The correct molecular formula could also be determined based on other RDB value information. In the current scenario, the resulting ions formed from electrospray ionization are evenelectron species, and if they contain a charge, then their bond saturation would result in a non-integer. As shown in Figure 8, only the top choice shows a value representing a protonated species, thus all other possible formulae could be eliminated. Finally, a comparison of experimental and theoretical isotopic distributions can also be used in structure elucidation. Figure 8 shows excellent agreement between the experimental and theoretical distribution for the $\mathrm{m} / \mathrm{z} 502$ metabolite.

Figure 9 displays the low mass range for the datadependent product ion spectrum from the metabolite with molecular ion at $m / z 502$, which is consistent with dihydroxylation of the parent nefazodone molecule. The base peak, which constitutes the ion at $\mathrm{m} / \mathrm{z} 290$ (mass error $=2.1 \mathrm{ppm})$, is consistent with addition of 16 Da to the ion at $m / z 274$ identical to that observed for the monohydroxy nefazodone metabolite $\mathrm{M} 8\left(\mathrm{~m} / \mathrm{z} 486, R_{\mathrm{t}}=\right.$ 


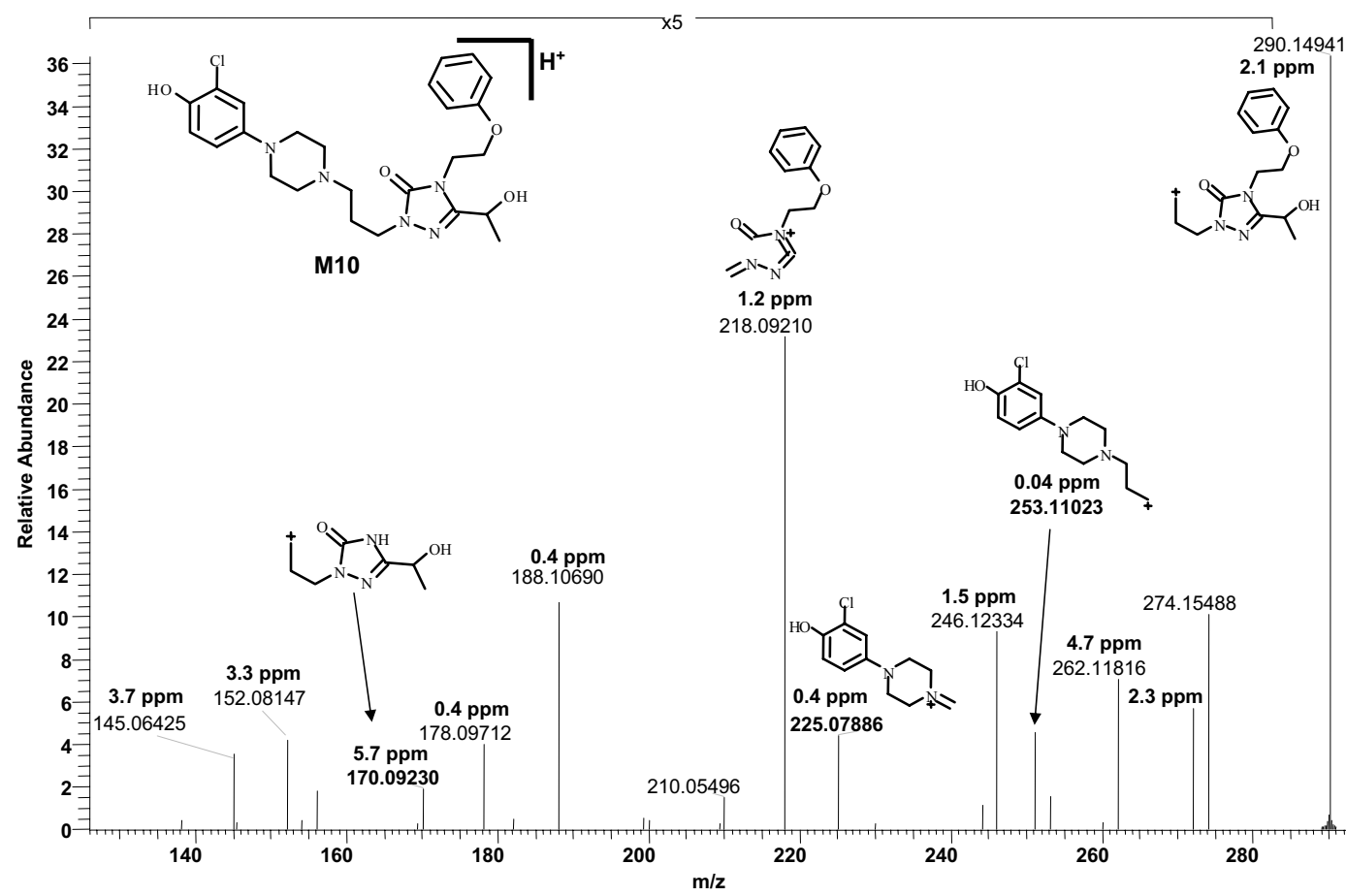

Figure 9. Full scan accurate mass product ion spectrum for the suspected dihydroxynefazodone metabolite M10 at $\mathrm{m} / \mathrm{z} 502$. The proposed structure of M10 is presented.

$12.25 \mathrm{~min})$. Based on this observation and the appearance of the fragment ions at $m / z 170,188,218,246$, and 262 (average mass error associated with these fragment ions is $2.7 \mathrm{ppm}$ ), we propose that one site of hydroxylation is the ethyl group on the triazolone ring as is noted with M8. Finally, the structures of the product ions at $\mathrm{m} / \mathrm{z} 225$ (mass error $=0.4 \mathrm{ppm}$ ) and 253 (mass error $=0.04 \mathrm{ppm}$ ) led us to propose 4-hydroxylation of the 3-chlorophenyl ring as a second site of hydroxylation considering that 4-hydroxynefazodone is the only metabolite detected from aromatic hydroxylation of nefazodone [14-17]. A similar conclusion was reached

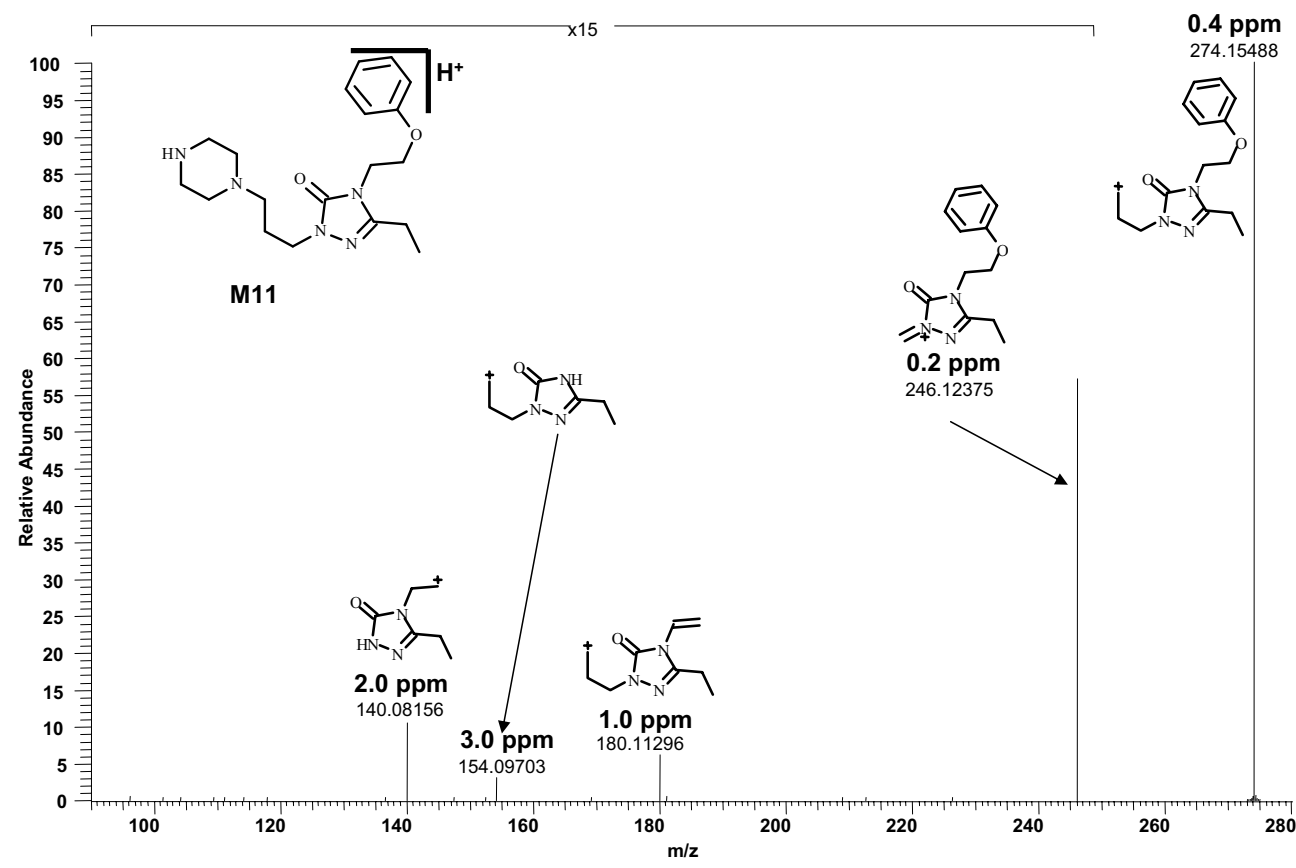

Figure 10. Full scan accurate mass product ion spectrum for the N-dephenylated piperazine metabolite M11. The proposed structure is presented. The formation of M11 is consistent with the mechanism proposed by Kalgutkar and coworkers [16]. 


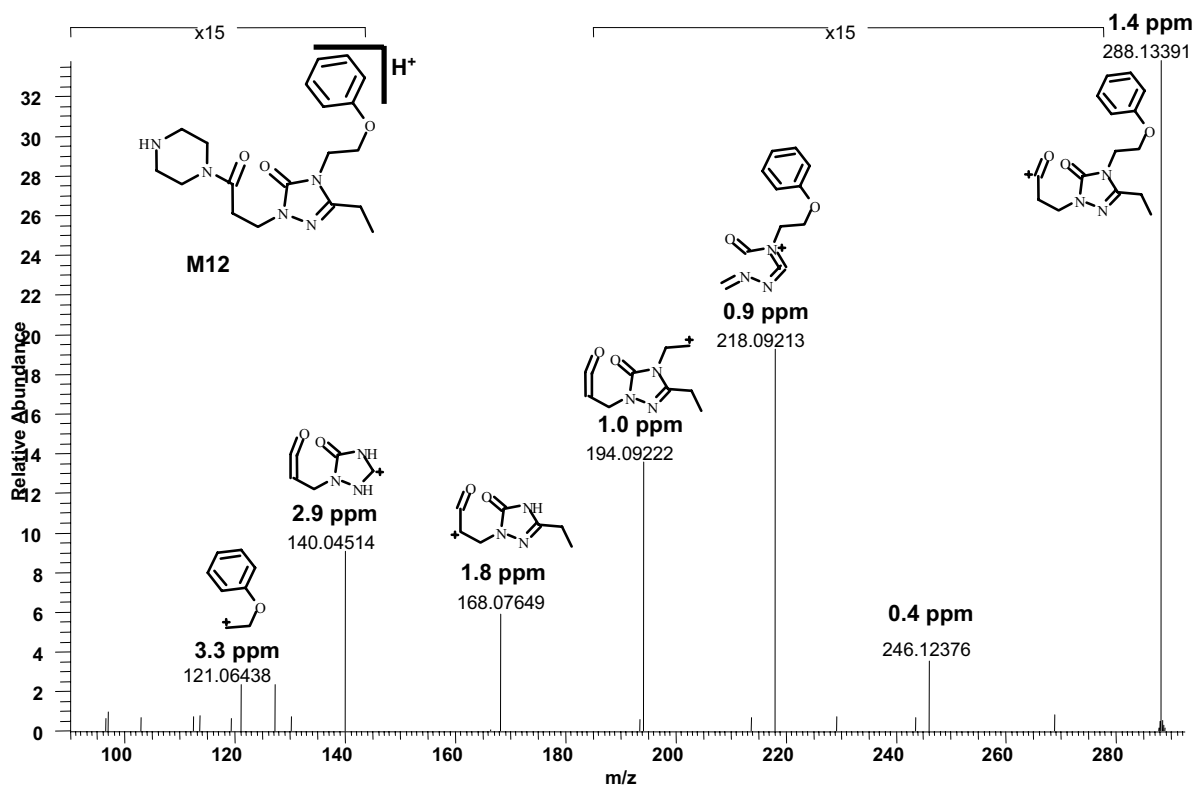

Figure 11. Full scan accurate mass product ion spectrum for the amide metabolite M12 obtained after further oxidation of M11.

Jemal and coworkers [17] who identified similar metabolic pathways for nefazodone.

Of much interest in the present context was the facile detection of the $\mathrm{N}$-dephenylated piperazine metabolites of nefazodone namely M11, M12, and M13, respectively, that have been previously reported [16]. Consistent with the loss of the 3-chlorophenyl moiety, there was a loss of the diagnostic chlorine isotopic pattern in the full scan spectra. In the present study, accurate mass full scan and MS/MS spectra were used to predict and verify the structures. Figure 10 shows the data-dependent MS/MS spectrum for the M11 metabolite. The predicted product ion structures and corresponding mass errors are also provided. All of the product ions of metabolite M11 as discerned from the CID spectrum are identical to those observed in the mass spectrum of parent nefazodone. Overall, the low mass errors associated with each of these product ions and the low mass error (1.1 ppm) observed in the full scan MS analysis of M11 increased confidence in the assigned metabolite structure.

Figures 11 and 12 show the product ion spectra for M12 and M13, respectively. M12 and M13 are derived from

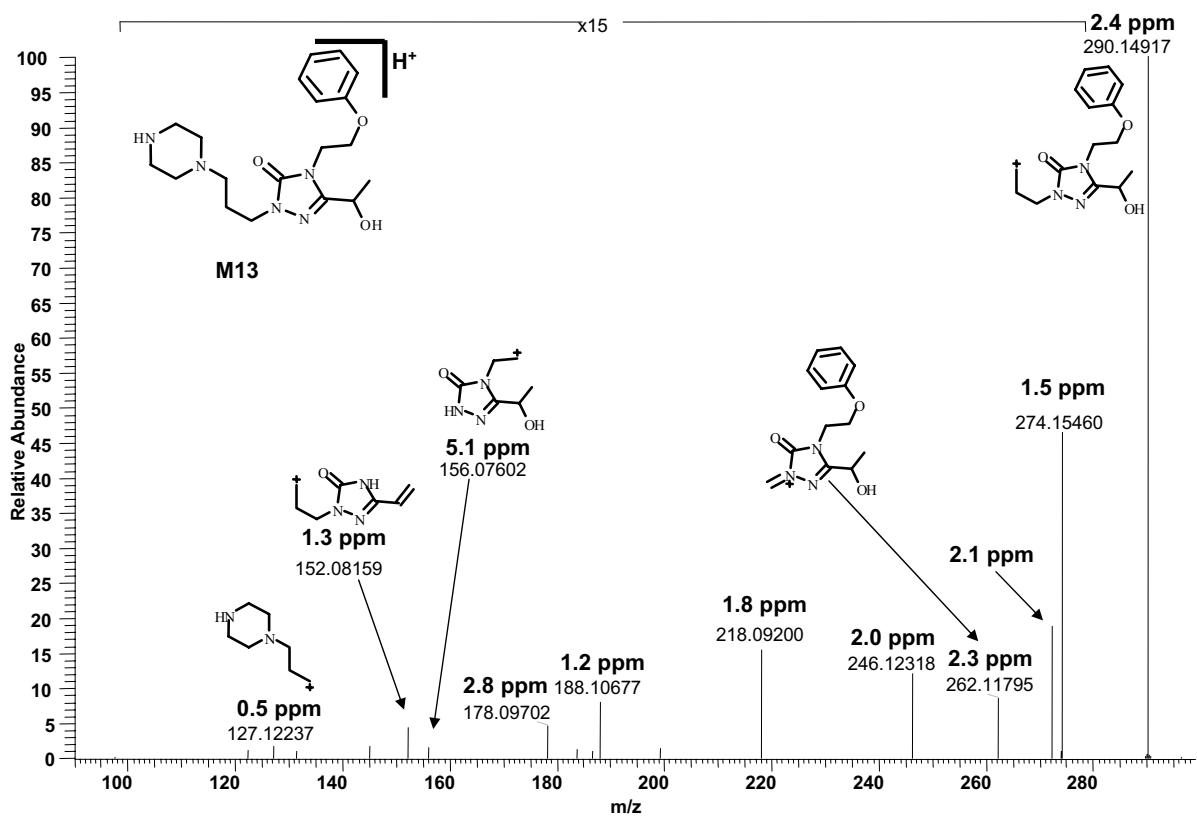

Figure 12. Full scan accurate mass product ion spectrum for M13 obtained via monohydroxylation of M11. 
Table 2. List of nefazodone and identified nefazodone metabolites from a $10 \mu \mathrm{M}$ incubation in human liver microsomes. Proposed chemical formulas used were obtained from Qual Browser based on nefazodone elemental composition and ranges. Mass accuracies were compared to the predicted chemical formula

\begin{tabular}{rccccccc}
\hline No. & Chemical formula & $R_{\mathrm{t}}$ (min) & Integrated peak area & Experimental mass & Theoretical mass & RDB & Mass error (ppm) \\
\hline \hline 1 & $\mathrm{C}_{10} \mathrm{H}_{13} \mathrm{O}_{1} \mathrm{~N}_{2} \mathrm{Cl}_{1}$ & 2.70 & $2.23 \mathrm{e} 5$ & 213.07921 & 213.07892 & 5.5 & 1.4 \\
2 & $\mathrm{C}_{19} \mathrm{H}_{30} \mathrm{O}_{3} \mathrm{~N}_{5}$ & 7.19 & $5.75 \mathrm{e} 6$ & 376.23505 & 376.23432 & 7.5 & 1.9 \\
3 & $\mathrm{C}_{10} \mathrm{H}_{13} \mathrm{~N}_{2} \mathrm{Cl}_{1}$ & 8.51 & $1.57 \mathrm{e} 6$ & 197.08452 & 197.08400 & 5.5 & 2.6 \\
4 & $\mathrm{C}_{19} \mathrm{H}_{30} \mathrm{O}_{2} \mathrm{~N}_{5}$ & 8.90 & $1.60 \mathrm{e} 7$ & 360.23981 & 360.23940 & 7.5 & 1.1 \\
5 & $\mathrm{C}_{15} \mathrm{H}_{20} \mathrm{O}_{2} \mathrm{~N}_{3}$ & 8.90 & $5.21 \mathrm{e} 5$ & 274.15567 & 274.15500 & 7.5 & 2.4 \\
6 & $\mathrm{C}_{19} \mathrm{H}_{28} \mathrm{O}_{3} \mathrm{~N}_{5}$ & 9.17 & $5.28 \mathrm{e} 6$ & 374.21829 & 374.21867 & 8.5 & -1.0 \\
7 & $\mathrm{C}_{25} \mathrm{H}_{33} \mathrm{O}_{4} \mathrm{~N}_{5} \mathrm{Cl}_{1}$ & 10.23 & $1.22 \mathrm{e} 7$ & 502.22086 & 502.22253 & 11.5 & -3.3 \\
8 & $\mathrm{C}_{15} \mathrm{H}_{21} \mathrm{O}_{3} \mathrm{~N}_{3}$ & 10.65 & $4.11 \mathrm{e} 6$ & 292.16608 & 292.16557 & 7.5 & 1.7 \\
9 & $\mathrm{C}_{15} \mathrm{H}_{19} \mathrm{O}_{4} \mathrm{~N}_{3}$ & 10.83 & $1.79 \mathrm{e} 6$ & 306.14557 & 306.14483 & 8.5 & 2.4 \\
10 & $\mathrm{C}_{15} \mathrm{H}_{19} \mathrm{O}_{4} \mathrm{~N}_{3}$ & 11.01 & $6.25 \mathrm{e} 5$ & 306.14539 & 306.14483 & 8.5 & 1.8 \\
11 & $\mathrm{C}_{25} \mathrm{H}_{32} \mathrm{O}_{3} \mathrm{~N}_{5} \mathrm{Cl}_{1}$ & 11.42 & $6.26 \mathrm{e} 6$ & 486.22742 & 486.22664 & 11.5 & 1.6 \\
12 & $\mathrm{C}_{23} \mathrm{H}_{28} \mathrm{O}_{3} \mathrm{~N}_{5} \mathrm{Cl}_{1}$ & 12.16 & $2.19 \mathrm{e} 6$ & 458.19559 & 458.19534 & 11.5 & 1.6 \\
13 & $\mathrm{C}_{25} \mathrm{H}_{32} \mathrm{O}_{3} \mathrm{~N}_{5} \mathrm{Cl}_{1}$ & 12.25 & $6.94 \mathrm{e} 6$ & 486.22748 & 486.22664 & 11.5 & 1.7 \\
14 & $\mathrm{C}_{25} \mathrm{H}_{32} \mathrm{O}_{2} \mathrm{~N}_{5} \mathrm{Cl}_{1}$ & 13.35 & $5.01 \mathrm{e} 7$ & 470.23224 & 470.23173 & 11.5 & 1.1 \\
15 & $\mathrm{C}_{25} \mathrm{H}_{31} \mathrm{O}_{3} \mathrm{~N}_{5} \mathrm{Cl}_{1}$ & 13.59 & $1.98 \mathrm{e} 5$ & 484.22167 & 484.21099 & 12.5 & 1.4 \\
16 & $\mathrm{C}_{25} \mathrm{H}_{32} \mathrm{O}_{3} \mathrm{~N}_{5} \mathrm{Cl}_{1}$ & 13.68 & $6.25 \mathrm{e} 5$ & 486.22754 & 486.22664 & 11.5 & 1.9 \\
\hline
\end{tabular}

further oxidative metabolism of M11. Comparison of the resulting product ion spectra with that shown in Figure 10 was used to determine the site of modification. For example, Figure 11 shows the primary fragment ion at $m / z 288$ (14 mass units greater than the $\mathrm{m} / \mathrm{z} 274$ product ion observed in Figure 10) is consistent with the formation of the acylium ion (mass error $=1.4 \mathrm{ppm}$ ) and suggestive of lack of modification to the piperazine ring. The observed product ions at $m / z 140,168$, and 194 is consistent with the site of oxidation residing on the propyl chain as opposed to either the 5-ethyltriazolone motif or the phenoxyethyl group. The observed acylium ion is consistent with P450catalyzed oxidation on the methylene group $\alpha$ to the tertiary piperazine nitrogen in M11 affording amide M12. Figure 12 shows the product ion spectrum for M13. The primary fragment ions used to determine the site of transformation were $\mathrm{m} / \mathrm{z} 127,152,156,262$, and 290 . The presence of the $\mathrm{m} / \mathrm{z} 127$ product ion indicates the piperazine ring was not the site of hydroxylation, rather the detection of the $m / z 156,262$, and 290 (average mass error $=2.1 \mathrm{ppm}$ ) show consistency with predicted structure involving the site of monohydroxylation on the 5-ethyltriazolone moiety.

Combining the results from full scan MS and MS/MS spectra provide a comprehensive list of nefazodone metabolites listed in Table 2 obtained from one LC-MS/ MS/MS experiment. The chemical formulas listed were predicted using Qual Browser and used for mass error determination. The average mass error for all 16 identified compounds was $1.8 \mathrm{ppm}$ despite a 20-fold difference in integrated peak areas. In addition, the RDB values calculated shows all values are related to the predicted structures increasing confidence in the predicted structures.

\section{Conclusions}

The present study outlines a facile method for identifying obvious and unanticipated metabolites in a single chromatographic run using accurate mass analysis of full scan MS and MS/MS spectra without the need for an inclusion list. The success of the methodology is based on fast scan speeds, high mass accuracy measurements with large intra-scan dynamic range, and the use of external calibration. Resulting mass spectral data can then be processed manually by experts in the biotransformation field or by predictive metabolite identification software to generate possible parent and product ion structures that are easily accepted or refuted using accurate mass analysis, bond saturation, and chemical formula ranges based on comparisons with mass spectral data on the parent drug. Combination of these features will undoubtedly improve confidence in the assignments of chemical formulae and biotransformation sites on metabolites. Furthermore, the ability to accurately measure product ion mass values without the need for internal calibrants or multiple experimental runs suggest that such analyses may be useful in early stages of drug discovery to screen the metabolic pattern of multiple compounds within a structural class or even structurally diverse chemical series in a rapid and iterative fashion and the knowledge of biotransformation pathways gained from such analysis could allow a facile optimization of ADME attributes. In vitro metabolite identification studies are currently underway on novel compounds with unknown biotransformation pathways and at concentrations close to typical therapeutic range $(0.1$ to $0.5 \mu \mathrm{M})$ to fully demonstrate the merits of this instrumentation.

\section{References}

1. Watt, A. P.; Mortishire-Smith, R. J.; Gerhard, U.; Thomas, S. R. Metabolite Identification in Drug Discovery. Curr. Opin. Drug Discov. Dev. 2003, 6(1), 57-65

2. Kalgutkar, A. S.; Soglia, J. R. Minimizing the Potential for Metabolic Activation in Drug Discovery. Exp. Opin. Drug Metab. Toxicol. 2005, 11 ), 91-141. 
3. Liu, D. Q.; Hop, C. E. C. A. Strategies for Characterization of Drug Metabolites Using Liquid Chromatography-Tandem Mass Spectrometry in Conjunction with Chemical Derivatization and On-Line H/D Exchange Approaches. I. Pharm. Biomed. Anal. 2005, 37(1), 1-18

4. Hop, C. E. C. A.; Yu, X.; Xu, X.; Singh, R.; Wong, B. K. Elucidation of Fragmentation Mechanisms Involving Transfer of Three Hydrogen Atoms Using a Quadrupole Time-of-Flight Mass Spectrometer. J. Mass Spectrom. 2001, 36(5), 575-579

5. Yin, W.; Doss, G. A.; Stearns, R. A.; Chaudhary, A. G.; Hop, C. E.; Frankin, R. B.; Kumar, S. A Novel P450-Catalyzed Transformation of the 2,2,6,6-Tetramethyl Piperidine Moiety to a 2,2-Dimethyl Pyrrolidine in Human Liver Microsomes: Characterization by High Resolution Quadrupole-Time-of-Flight Mass Spectrometry and 1H-NMR. Drug Metab. Dispos. 2003, 31(2), 215-223

6. Gangl, E.; Utkin, I.; Gerber, N.; Vouros, P. Structural Elucidation of Metabolites of Ritonavir and Indinavir by Liquid ChromatographyMass Spectrometry. J. Chromatogr. A. 2002, 974(1/2), 91-101

7. Mutlib, A. E.; Shockcor, J. P. 2003; Application of LC/MS, LC/NMR, NMR and Stable Isotopes in Identifying and Characterizing Metabolites. Lee, J. S.; Obach, R. S.; Fisher, M. B., Eds.; In Drug Metabolizing Enzymes: Cytochrome P450 and Other Enzymes in Drug Discovery and Development; p 33Marcel Dekker: New York, NY, Fontis Media: Lausanne, Switzerland.

8. Li, L.; Chiarelli, M. P.; Branco, P. S.; Marques, M. M.; Goncalves, L. L.; Beland, F. A. Differentiation of Isomeric C8-Substituted Alkylaniline Adducts of Guanine by Electrospray Ionization and Tandem Quadrupole Ion Trap Mass Spectrometry. J. Am. Soc. Mass Spectrom. 2003, 14(12), 1488-1492

9. Hopfgartner, G.; Chernushevich, I. V.; Covey, T.; Plomley, J. B.; Bonner, R. Exact Mass Measurement of Product Ions for the Structural Elucidation of Drug Metabolites with a Tandem Quadrupole OrthogonalAcceleration Time-of-Flight Mass Spectrometer. J. Am. Soc. Mass Spectrom. 1999, 10(12), 1305-1314

10. Deroussent, A.; Re, M.; Hoellinger, H.; Vanquelef, E.; Duval, O.; Sonnier, M.; Cresteil, T. In Vitro Metabolism of Ethoxidine by Human CYP1A1 and Rat Microsomes: Identification of Metabolites by HighPerformance Liquid Chromatography Combined with Electrospray Tandem Mass Spectrometry and Accurate Mass Measurements by Time-of-Flight Mass Spectrometry. Rapid Commun. Mass Spectrom. 2004, 18(4), 474-482

11. Castro-Perez, J.; Plumb, R.; Granger, J. H.; Beattie, I.; Joncour, K.; Wright, A. Increasing Throughput and Information Content for in Vitro Drug Metabolism Experiments Using Ultra-Performance Liquid Chro- matography Coupled to a Quadrupole Time-of-Flight Mass Spectrometer. Rapid Commun. Mass Spectrom. 2005, 19(6), 843-848

12. Hardman, M.; Makarov, A. A. Interfacing the Orbitrap Mass Analyzer to an Electrospray Ion Source. Anal. Chem. 2003, 75(7), 1699-1705

13. Thevis, M.; Makarov, A. A.; Horning, S.; Schanzer, W. Mass Spectrometry of Stanozolol and Its Analogs Using Electrospray Ionization and Collision-Induced Dissociation with Quadrupole-Linear Ion Trap and Linear Ion Trap-Orbitrap Hybrid Mass Snalyzers. Rapid Commun. Mass Spectrom. 2005, 19(22), 3369-3378

14. Mayol, R. F.; Cole, C. A.; Luke, G. M.; Colson, K. L.; Kerns, E. H. Characterization of the Metabolites of the Antidepressant Drug Nefazodone in Human Urine and Plasma. Drug Metab. Dispos. 1994, 22(2), 304-311

15. Von Moltke, L. L.; Greenblatt, D. J.; Granda, B. W.; Grassi, J. M.; Schmider, J.; Harmatz, J. S.; Shader, R. I. Nefazodone, Meta-Chlorophenylpiperazine, and their Metabolites in Vitro: Cytochromes Mediating Transformation, and P450-3A4 Inhibitory Actions. Psychopharmacology 1999, 145(1), 113-122.

16. Kalgutkar, A. S.; Vaz, A. D. N.; Lame, M. E.; Henne, K. R.; Soglia, J.; Zhao, S. X.; Abramov, Y. A.; Lombardo, F.; Collin, C.; Hendsch, Z. S.; Hop, C. E. C. A. Bioactivation of the Nontricyclic Antidepressant Nefazodone to a Reactive Quinone-Imine Species in Human Liver Microsomes and Recombinant Cytochrome P450 3A4. Drug Metab. Dispos. 2005, 33(2), 243-253

17. Jemal, M.; Ouyang, Z.; Zhao, W.; Zhu, M.; Wu, W. W. A Strategy for Metabolite Identification Using Triple-Quadrupole Mass Spectrometry with Enhanced Resolution and Accurate Mass Capability. Rapid Commun. Mass Spectrom. 2003, 17(24), 2732-2740

18. Hu, Q.; Noll, R. J.; Li, H.; Makarov, A.; Hardman, M.; Cooks, R. G. The Orbitrap: A new Mass Spectrometer. J. Mass Spectrom. 2005, 40(4), 430-443

19. McLafferty, F. W.; Turecek, F. Interpretation of Mass Spectra, 4th ed.; University Science Books: Mill Valley, CA, 1993, p 27.

20. Pittenauer, E.; Zehl, M.; Mistrik, R.; Allmaier, G. Evaluation of ESI-MSn Ion Trap and MALDI-MSn Ion Trap/Reflectron TOF Mass Spectra $(\mathrm{n}=1-3)$ for Incorporation into a Searchable Database. Proceedings of the 52nd ASMS Conference on Mass Spectrometry and Allied Topics; Nashville, TN 2004.

21. Mistrik, R. Comprehensive Database of Mechanisms of Peptide Fragmentation: A Key Step Towards Confident Identification of Proteins Proceedings of the 52nd ASMS Conference on Mass Spectrometry and Allied Topics; Nashville, TN 2004. 